Ann. Génét. Sél. anim., 1982, 14 (2), 135-160

I.N.R.A. - C.N.R.Z.

Département de Génétique Animale BIBLIOTHEOUE

F 78350 JOUY EN JOSAS

\title{
Sex linked dwarf gene (dw) in White Leghorn laying hens under normal or hot temperature
}

\author{
A.K. BANERJEE (*), A. BORDAS and P. MÉRAT \\ I.N.R.A., Laboratoire de Génétique factorielle, \\ Centre de Recherches zootechniques, F 78350 Jouy-en-Josas
}

\begin{abstract}
Summary
White Leghorn females from 7 sire families segregating at the sex-linked dwarf locus (Dw and $\mathrm{dw}$ ) were studied in individual cages at moderate $\left(13-23^{\circ} \mathrm{C}\right)$ and high $\left(25-34^{\circ} \mathrm{C}\right)$ temperature from 18 to 39 weeks of age. Traits concerning body weight, feed efficiency, egg production to 39 th week of age, egg quality along with plasma uric acid and glucose at 18 weeks were measured. In heated group plasma uric acid at 39 weeks, dry matter and moisture in faeces along with daily water intake were also recorded. Results are as follows :
\end{abstract}

1. Adult body weight in dwarfs was 38 p. 100 lower than that of normals in control and 32 p. 100 in heated group whereas food consumption of dwarfs was respectively 36 and 37 p. 100 less than of normals in control and in heated group.

2. Reduction of egg number from age at first egg to 39th week of age in dwarfs was 20 and 41 p. 100 of normals in control and heated group respectively. Total egg mass per 28 days in dwarfs was reduced by 34 p. 100 as compared to normals in control group and by $37 \mathrm{p} .100$ in heated group. dw/Dw egg weight ratio increased from 0.85 to 0.89 from 27th to 39th week of age. During the first 7 weeks of laying in control group p. 100 double yolk, soft and broken eggs were highly significantly lower in dwarfs; in both groups clutch size was significantly lower, but days of laying pauses were not significantly higher.

3. Age at sexual maturity in dwarfs was only one day later than for normals (156 v. 155) in control group while in heated group and unusual delay of sexual maturity in both normal and dwarfs was observed which appears to be due to an effect other than heat (201 and 182 days for dwarfs and normals respectively).

4. Egg shell thickness was lower for $d w$ hens in both environments, the difference being higher and highly significant in heated group.

5. A very highly significant effect of genotype was observed in rectal temperature in both control and heated group, dwarf having a lower rectal temperature than normals.

6. A very significant effect of genotype and sire family was observed in plasma uric acid measured at 39 weeks in heated group. The difference between genotypes was in the same direction (higher value for dwarfs) but comparatively less than that observed at 18 weeks. Mean level of plasma uric acid at 39 weeks was increased compared to that at 18 weeks.

7. Phenotypic correlations of egg shell traits with body weight, observed food consumption, egg mass, egg number and clutch size were all positive and mostly significant in dwarfs while they are unsignificant and generally negative in $\mathrm{Dw}$ genotype.

(*) Permanent adress : Department of Animal Science, College of Agriculture, G.-B. Pant University of Agriculture \& Technology, PANTNAGAR - 263145, India. 
8. In general plasma uric acid at 18 weeks and at 39 weeks was negatively associated with egg number, egg mass and clutch size. These correlations were more pronounced in control group for Dw genotype.

9. The especially large reduction of body weight and laying performance and the lowering of egg shell traits by the dw gene may correspond to the small size of the Leghorn stock used in the present study.

\section{Introduction}

Utilization of the sex linked dwarf gene in layer populations appears to be a subject of controversy. FRENCH \& NoRDSKOG (1973) state that better feed efficiency should be searched by selection for low body weight in conventional hens whereas BERNIER \& ARSCOTT (1972) think that improvements already obtained justify the pursuit of selection of $\mathrm{dw}$ "mini birds ». MÉrat et al. (1974), Selvarajah (1974) and Horst \& Petersen (1978) suggest that dwarf hens can be at an advantage in hot climates. From this point of view, BANERJEE et al. (1981) observed that studies are needed on the effect of the dwarf gene on feed efficiency, egg production, egg and physiological traits in layer populations reared from the beginning under hot environmental conditions. The realization of such conditions represents part of the present paper, the main purpose of it being to evaluate effects of the dw gene on production and physiological traits of laying hens in a small-sized Leghorn strain.

\section{Material and methods}

\section{1. - Experimental birds and conditions}

The birds used and the experimental conditions up to the age of 18 weeks have been described earlier (BANERJEE et al., 1981). We shall recall the main points which are useful for the understanding of the present paper. Dwarf and normal White Leghorn females were obtained from 7 heterozygous sires $(D w d w)$ mated with dwarf females. Two hatches were obtained at a 2 weeks interval in September, 1980. The chicks were reared on deep litter till 18 weeks of age, the first hatch under fluctuating normal temperature (between 15 and $20^{\circ} \mathrm{C}$ ) after 4 weeks of age, the second under higher temperature (between 25 and $30^{\circ} \mathrm{C}$ ). The first hatch corresponded to the «control» group and the second to the «heated» group. After 18 weeks of age 249 Whice Leghorn females comprising 138 (66 Dw ; $72 \mathrm{dw}$ ) individuals in control group and $111(61 \mathrm{Dw} ; 50 \mathrm{dw})$ in heated group, were transferred to individual laying cages contained in two separate houses. The control group was maintained under normal temperature with an average daily minimum and maximum of 17.4 and $18.3{ }^{\circ} \mathrm{C}$ respectively ; the heated group was kept at hot temperature with 27.6 and $31.1^{\circ} \mathrm{C}$ as average daily minimum and maximum. The experimental period lasted till 39 weeks of age for both groups. During this period the birds received 14 hours of light and 10 hours of darkness per day. Water and feed were given ad libitum to both groups. The feed contained $16 \mathrm{p}$. 100 total protein and approximately $2600 \mathrm{Kcal} / \mathrm{kg} \mathrm{M.E.} \mathrm{and} 3.5$ p. 100 calcium. 
It must be added that, between the two experimental groups, additional environmental differences other than ambient temperature cannot be ruled out. Difference in day of hatch can be considered as a minor source of variation for the traits recorded here. A more important factor is the presence of mortality, mainly by Marek's disease as this stock had not been vaccinated. This mortality was considerably higher in the heated than in control group. (From 18 to 39 weeks of age, percent mortality in the control group was respectively 6.1 and 21.2 for Dw and dw females; the corresponding figures in the heated group are 27.5 and 28.2).

\section{2. - Traits measured or calculated}

Only birds surviving beyond the end of test period are considered.

Traits measured or calculated during the experimental period between 18 th and 39 th week of age, symbols used and their sequence in the succeeding tables are listed in table 2. Data on egg shell, double yolks, soft eggs and broken eggs, blood spots in eggs, dry matter and moisture in feces, are presented in percentage. On the other hand, the data on 18 week body weight, plasma uric acid and glucose at 18 weeks have been reproduced from the previous article (BANERJEE et al., 1981). As table 2 shows, a few variables were recorded only in control group, because their measurement was not possible or not satisfactory because of low variation in the heated group owing to delayed sexual maturity.

Some other traits (plasma uric acid, water intake, water and dry matter in feces) were measured only in heated group. Concerning the trait « residual food consumption », the procedure used for obtaining it is as follows : during several periods of 28 days each ( 3 for control, 2 for heated group), the per bird per period observed food consumption $(\mathrm{O})$, mean body weight $(\mathrm{W})$ for each period, body weight variation between the start and end of the period $(\Delta W)$ and total weight of eggs laid (E) were recorded. For each individual a mean value of the three or resp. two periods for the above characters was obtained to calculate the predicted feed intake $(\mathrm{T})$ by a multiple regression equation :

$$
\mathrm{T}=\mathrm{a} \mathrm{W}^{a}+\mathrm{b} \Delta \mathrm{W}+\mathrm{cE}
$$

This equation has been described by BYERLY (1941) and utililized by Lesson et al. (1973), Gous et al. (1978), Mc Donald (1978), Bordas \& Mérat (1981). The optimum value of $\alpha$ in the present study has been taken as 0.5 with the purpose of giving minimum error variance to the regression. For each of the control and heated groups one regression equation was established after verifying that in each group, the coefficients $a, b$, c were not significantly different for the Dw and $d w$ genotypes.

The utilized equations are :

$$
\begin{aligned}
& \mathrm{T}=93.87 \mathrm{~W}^{0.5}+1.197 \Delta \mathrm{W}+0.760 \mathrm{E} \text { (Control group) } \\
& \mathrm{T}=58.21 \mathrm{~W}^{0.5}+0.915 \Delta \mathrm{W}+1.211 \mathrm{E} \text { (Heated group) }
\end{aligned}
$$

The difference between observed and predicted feed intake $(\mathrm{O}-\mathrm{T})$ for each individual is taken as residual feed consumption (R). 


\section{3. - Statistical analysis}

For each mesure, a within group two factor analysis of variance was done, sources of variation being Genotype, sire Family and Genotype $\times$ Family interaction. A three factor analysis of variance with between groups component of variation was not carried out as variation between groups caused by factors other than temperature mainly associated with Marek's disease (see above) was suspected. Within group within genotype phenotypic correlations between characters were obtained. Correlations with the two genotypes combined (on a within genotype basis) were calculated after testing correlations for homogenity between genotypes. Correlations with genotypes pooled were tested, on the other hand, for homogeneity between treatments, and conversely a pooled estimate (treatments grouped on a within treatment basis) for each genotype was carried out for correlations which revealed a between genotypes heterogeneity.

\section{Results}

Table 1 presents the average and range of maximum and minimum temperature $\left({ }^{\circ} \mathrm{C}\right)$ for control and heated group during the experimental period. The means of the two genotypes and the percent of dwarf to normal genotype for each character measured under control and heated group are presented in table 3. The two factor (genotype, family) analyses of variance within group, for the same characters are given in table 4. Within genotype within group phenotypic correlations between 20 of the characters measured for control group and 22 for heated group were estimated. However, in tables 5 and 6 corresponding respectively to control and heated group, the correlation value for each genotype is given only when there is significant heterogeneity between these genotypes; in other cases a common value (on a within genotype basis) is presented. In addition, for each genotype a pooled estimate across environments was calculated when no statistical heterogeneity between treatments appeared. The result is presented in table 7, only for correlations differing significantly on the whole for Dw and dw birds.

\section{TABLE 1}

Averages and range of daily maximum and minimum temperature $\left({ }^{\circ} \mathrm{C}\right)$ during the period 18-39 weeks of age.

Moyenne et intervalle de variation de la température journalière maximale et minimale $\left({ }^{\circ} \mathrm{C}\right)$ entre 18 et 39 semaines d'âge.

\begin{tabular}{c|c|c|c|c}
\hline \multirow{2}{*}{ Temperature } & \multicolumn{4}{|c}{ Treatment } \\
\cline { 3 - 5 } & \multicolumn{2}{|c|}{ Control } & \multicolumn{2}{c}{ Heated } \\
\cline { 2 - 5 } & Average & Range & Average & Range \\
\hline Maximum $\ldots \ldots \ldots \ldots$ & 18.3 & $14.2-23.3$ & 31.1 & $29.5-33.5$ \\
Minimum $\ldots \ldots \ldots \ldots .6$ & 17.4 & $12.6-22.4$ & 27.6 & $24.8-29.4$ \\
\hline \hline
\end{tabular}




\section{TABLE 2}

Symbols and description of traits.

Symboles et définition des critères.

\begin{tabular}{|c|c|c|}
\hline No. & Symbol & Description \\
\hline 1 & $\mathbf{W} 2$ & $\begin{array}{l}\text { Variables connected with food efficiency on } 28 \mathrm{~d} \text {. periods } \\
\text { Body weight }(\mathrm{g}) \text { - based on mean of weights at start and end of each } \\
28 \text { day period. }\end{array}$ \\
\hline 2 & $\Delta \mathrm{W}$ & $\begin{array}{l}\text { Variation in body weight (g) between start and end of each } 28 \text { day } \\
\text { period. }\end{array}$ \\
\hline 3 & $\mathbf{E}$ & Total weight $(\mathrm{g})$ of eggs laid / 28 days. \\
\hline 4 & $\mathbf{O}$ & Observed feed consumption $(\mathrm{g}) / 28$ days. \\
\hline 5 & $\mathbf{R}$ & $\begin{array}{l}\text { Deviation }(\mathrm{g}) \text { of observed feed intake from predicted feed intake } \\
\text { from regression on body weight, egg mass and variation of } \\
\text { body weight } / 28 \text { days. }\end{array}$ \\
\hline 6 & *W1 & $\begin{array}{l}\text { Variables concerning body weight, egg production and egg traits } \\
\text { Body weight }(\mathrm{g}) \text { at } 18 \text { weeks of age. }\end{array}$ \\
\hline 7 & $\Delta \mathbf{W}^{\prime}$ & Variation in body weight $(\mathrm{g})$ between 18 and 27 weeks of age. \\
\hline 8 & ASM & Age at 1st egg (days). \\
\hline 9 & EN & Total egg number from 1st egg to end of test period. \\
\hline 10 & cEW1 & Mean egg weight $(\mathrm{g})$, first 15 days of test period. \\
\hline 11 & EW2 & Egg weight (g), last 15 days of test period. \\
\hline 12 & $\% \mathrm{ES}$ & \begin{tabular}{c|l}
$\begin{array}{c}\text { Percent egg shell with } \\
\text { membrane. }\end{array}$ & $\begin{array}{l}\text { On one egg per bird, during 28th week } \\
\text { of age for control and 38th week of age } \\
\text { for heated group due to time limitations. }\end{array}$ \\
Yolk-Albumen ratio.
\end{tabular} \\
\hline 14 & $\mathrm{AH}$ & $\begin{array}{l}\text { Albumen height }(1 / 10 \mathrm{~mm} \text {; mean of eggs laid during the last } \\
18 \text { days of test period). }\end{array}$ \\
\hline 15 & EST & $\begin{array}{l}\text { Egg shell thickness including membrane }(0.01 \mathrm{~mm} \text {; mean of eggs } \\
\text { laid during the last } 18 \text { days of test period). }\end{array}$ \\
\hline 16 & cDYE & Percent double yolk eggs from first egg till 27 weeks of age. \\
\hline 17 & c\% SE & Percent soft-shelled eggs from first egg till 27 weeks of age. \\
\hline 18 & c\% BE1 & Percent broken eggs from first egg till 27 weeks of age. \\
\hline 19 & $\% \mathrm{BE} 2$ & Percent broken eggs during test period. \\
\hline 20 & c\% BS & Percent blood or meat spots on 3 eggs/bird. \\
\hline 21 & $\mathrm{CS}$ & Mean clutch size during test period. \\
\hline
\end{tabular}


TABLE 2 (continued)

\begin{tabular}{|c|c|c|}
\hline No. & Symbol & Description \\
\hline 22 & EP & Total days of pauses of more than 2 days during test period. \\
\hline 23 & NEP & Number of laying pauses of more than 2 days during test period. \\
\hline & & Morphological or physiological variables \\
\hline 24 & WL & Wattle length $(\mathrm{mm})$ at the end of test period. \\
\hline 25 & SL & Shank length $(\mathrm{mm})$ at the end of test period. \\
\hline 26 & ST & Shank thickness $(0.1 \mathrm{~mm})$ at the end of test period. \\
\hline 27 & $\mathrm{Rt}^{\mathrm{o}}$ & $\begin{array}{l}\text { Rectal temperature }\left({ }^{\circ} \mathrm{C}\right) \text { at the end of test period (between } 9.00 \text { and } \\
10.30 \text { a.m.). }\end{array}$ \\
\hline 28 & *PUA1 & $\begin{array}{l}\text { Plasma uric acid }(\mathrm{mg} / \mathrm{l}) \text { at } 18 \text { weeks (blood collection between } \\
9.00 \text { a.m. and } 12.00) \text {. }\end{array}$ \\
\hline 29 & *PG & $\begin{array}{l}\text { Plasma glucose }(\mathrm{mg} / 100 \mathrm{ml}) \text { at } 18 \text { weeks (blood collection between } \\
9.00 \text { a.m. and } 12.00) .\end{array}$ \\
\hline 30 & hPUA2 & $\begin{array}{l}\text { Plasma uric acid }(\mathrm{mg} / \mathrm{l}) \text { at the end of test period (blood collection } \\
\text { between } 9.00 \text { a.m. and } 12.00) \text {. }\end{array}$ \\
\hline 31 & hWI & Daily water intake $(\mathrm{ml})$ during the last 18 days of the test period. \\
\hline 32 & hDMF & $\begin{array}{l}\text { Dry matter }(\mathrm{g}) \text { in feces (traits } 32 \text { and } 33 \text { on faeces collected during } \\
48 \text { hours within the last } 28 \text {-day period). }\end{array}$ \\
\hline 33 & $\mathrm{~h} \% \mathrm{MF}$ & Percent moisture in faeces. \\
\hline
\end{tabular}

Note :

1. For control group, traits no. 1, 2, 3, 4, 5, 19, 21, 22, 23 were recorded during three 28-day periods beginning from 27 weeks of age. In heated group the 1st 28-day period was not included in calculation except for characters no. $21,22,23$.

2. Data are on individual basis (those collected over the whole test period are an average of the three $28 \mathrm{~d}$. periods for control, of the two periods for heated group).

3. $c=$ Characters measured only in control group.

4. $\mathrm{h}=$ Characters measured only in heated group.

5. * Data reproduced from BanERJEe et al., 1981. 
TABLE 3

Averages of parameters concerning egg production, feed efficiency, egg traits, anatomical and physiological traits.

Moyennes des paramètres concernant la production d'œufs, l'efficacité alimentaire, les caractéristiques des oufs, des caractères anatomiques et physiologiques.

\begin{tabular}{|c|c|c|c|c|c|c|}
\hline \multirow{3}{*}{ Paramet. } & \multicolumn{3}{|c|}{ Control group } & \multicolumn{3}{|c|}{ Heated group } \\
\hline & \multicolumn{2}{|c|}{ Genotype } & \multirow{2}{*}{$\frac{\mathrm{dw}}{\mathrm{Dw}} \times 100$} & \multicolumn{2}{|c|}{ Genotype } & \multirow{2}{*}{$\frac{\mathrm{dw}}{\mathrm{Dw}} \times 100$} \\
\hline & Dw & $d w$ & & Dw & $d w$ & \\
\hline W2 & 1533.0 & 944.0 & 61.6 & 1424.0 & 968.4 & 68.0 \\
\hline$\Delta \mathrm{W}$ & 8.4 & 2.2 & - & -6.9 & -38.2 & - \\
\hline$E$ & 1106.2 & 732.2 & 66.2 & 1043.8 & 652.9 & 62.5 \\
\hline $\mathrm{O}$ & 3090.0 & 1986.0 & 64.3 & 2573.2 & 1625.0 & 63.1 \\
\hline $\mathbf{R}$ & 11.4 & -10.4 & - & 26.4 & -33.8 & - \\
\hline W1 & 1337.5 & 849.5 & 63.5 & 1272.1 & 851.2 & 66.9 \\
\hline$\Delta \mathbf{W}^{\prime}$ & 185.7 & 95.8 & 51.6 & 169.1 & 178.2 & 105.4 \\
\hline ASM & 155.0 & 156.1 & 100.7 & 182.0 & 200.9 & 110.4 \\
\hline EN & 104.5 & 83.4 & 79.8 & 65.0 & 38.4 & 59.1 \\
\hline EW1 & 47.3 & 40.6 & 85.8 & - & - & - \\
\hline EW2 & 50.9 & 45.5 & 89.3 & 50.8 & 44.8 & 88.1 \\
\hline$\% \mathrm{ES}$ & 9.4 & 9.5 & 101.3 & 9.7 & 9.6 & 98.8 \\
\hline $\mathrm{Y} / \mathrm{A}$ & 41.8 & 42.6 & 101.9 & 45.9 & 40.8 & 88.8 \\
\hline $\mathrm{AH}$ & 86.1 & 83.3 & 96.7 & 85.1 & 90.6 & 106.5 \\
\hline EST & 37.3 & 36.4 & 97.6 & 37.9 & 35.7 & 94.2 \\
\hline$\%$ DYE & 8.2 & 1.2 & 14.3 & - & - & - \\
\hline$\% \mathrm{SE}$ & 3.7 & 0.5 & 12.3 & 一 & - & 一 \\
\hline$\% \mathrm{BE} 1$ & 4.8 & 1.2 & 25.7 & 一 & - & - \\
\hline$\%$ BE2 & 2.0 & 1.8 & 90.6 & 5.3 & 7.9 & 149.0 \\
\hline$\% \mathrm{BS}$ & 0.4 & 0.1 & 28.9 & 一 & - & - \\
\hline $\mathrm{CS}$ & 9.2 & 2.5 & 27.6 & 3.2 & 1.4 & 43.8 \\
\hline EP & 6.3 & 12.3 & 196.3 & 3.4 & 4.9 & 142.7 \\
\hline NEP & 0.4 & 0.5 & 142.1 & 0.5 & 0.8 & 160.4 \\
\hline WL & 35.6 & 29.7 & 83.4 & 35.7 & 31.9 & 89.4 \\
\hline SL & 100.7 & 80.6 & 80.0 & 105.4 & 83.5 & 79.2 \\
\hline ST & 78.1 & 68.8 & 88.1 & 77.0 & 70.4 & 91.4 \\
\hline $\mathrm{Rt}^{\mathrm{o}}$ & 40.6 & 40.3 & 99.3 & 40.7 & 40.4 & 99.3 \\
\hline PUA1 & 19.7 & 36.5 & 184.6 & 32.6 & 52.2 & 160.2 \\
\hline PG & 223.9 & 215.6 & 96.3 & 219.3 & 211.8 & 96.6 \\
\hline PUA2 & - & 一 & - & 52.7 & 67.7 & 128.5 \\
\hline WI & - & 一 & - & 277.1 & 192.3 & 69.4 \\
\hline DMF & - & - & - & 53.1 & 38.1 & 71.8 \\
\hline$\% \mathrm{MF}$ & - & 一 & - & 66.9 & 63.3 & 94.6 \\
\hline
\end{tabular}


TABLE 4

Analysis of variance concerning egg production, feed efficiency, egg traits, anatomical and physiological traits.

Analyse de variance concernant la production d'œufs, l'efficacité alimentaire, les caractéristiques des aufs, les caractères anatomiques et physiologiques.

\begin{tabular}{|c|c|c|c|c|c|c|c|c|}
\hline \multirow{3}{*}{$\begin{array}{l}\text { Para- } \\
\text { met. }\end{array}$} & \multicolumn{4}{|c|}{ Control group } & \multicolumn{4}{|c|}{ Heated group } \\
\hline & \multicolumn{3}{|c|}{$F$ ratio } & \multirow{2}{*}{$\begin{array}{l}\text { d.f. } \\
\text { resi- } \\
\text { dual }\end{array}$} & \multicolumn{3}{|c|}{$F$ ratio } & \multirow{2}{*}{$\begin{array}{l}\text { d.f. } \\
\text { resi- } \\
\text { dual }\end{array}$} \\
\hline & $\begin{array}{c}\text { Genotype } \\
(\mathrm{df}=1)\end{array}$ & $\begin{array}{c}\text { Family } \\
(\mathrm{df}=6)\end{array}$ & $\begin{array}{c}\text { Inter- } \\
\text { action } \\
(\mathrm{df}=6)\end{array}$ & & $\begin{array}{l}\text { Genotype } \\
(\mathrm{df}=1)\end{array}$ & $\begin{array}{c}\text { Family } \\
(\mathrm{df}=6)\end{array}$ & $\begin{array}{c}\text { Inter- } \\
\text { action } \\
(\mathrm{df}=6)\end{array}$ & \\
\hline W2 & $609.8^{* * * *}$ & $3.0^{* * *}$ & 1.9 & 104 & $197.7 * * *$ & $4.6 * * *$ & 0.5 & 62 \\
\hline$\Delta \mathrm{W}$ & 1.3 & 0.3 & 0.5 & 104 & $11.5 * * *$ & $7.4 * * *$ & 0.7 & 62 \\
\hline E & $52.7 * * *$ & 0.9 & 1.7 & 104 & $117.3 * * *$ & $3.1 * *$ & 1.2 & 62 \\
\hline $\mathrm{O}$ & $330.9 * * *$ & $2.5 *$ & 1.4 & 104 & $226.2 * * *$ & $3.3^{* *}$ & 0.7 & 62 \\
\hline $\mathbf{R}$ & 1.2 & $3.1 * *$ & 0.9 & 104 & $4.1 *$ & $2.5^{*}$ & $2.6^{*}$ & 62 \\
\hline W1 & $636.9 * * *$ & $3.0 * *$ & 0.9 & 104 & $307.7 * * *$ & $3.7 * * *$ & 0.1 & 62 \\
\hline$\Delta \mathbf{W}^{\prime}$ & $43.5 * * *$ & 1.8 & 1.4 & 104 & 0.2 & 0.5 & 1.1 & 62 \\
\hline ASM & 0.4 & $3.7 * *$ & 1.9 & 104 & $28.5 * * *$ & 0.9 & 1.6 & 62 \\
\hline EN & $22.9 * * *$ & 0.6 & 1.9 & 104 & $72.1 * * *$ & 0.9 & 2.0 & 62 \\
\hline EW1 & $166.7 * * *$ & $4.5 * * *$ & $2.5 *$ & 101 & 一 & - & - & - \\
\hline EW2 & $53.9 * * *$ & 2.0 & 1.1 & 92 & $66.9 * * *$ & $2.3 *$ & 2.2 & 62 \\
\hline$\% \mathrm{ES}$ & 1.5 & $4.3 * * *$ & 1.4 & 104 & 1.3 & 2.1 & 1.0 & 59 \\
\hline $\mathbf{Y} / \mathbf{A}$ & 0.0 & 0.1 & 0.4 & 104 & $38.8 * * *$ & 1.1 & $3.4^{* *}$ & 59 \\
\hline AH & $4.6^{*}$ & $2.5 *$ & 1.3 & 99 & $16.4 * * *$ & 0.7 & 2.1 & 61 \\
\hline EST & $3.9 *$ & $2.4 *$ & 1.2 & 99 & $28.8 * * *$ & $2.4 *$ & $2.4^{*}$ & 61 \\
\hline$\%$ DYE & $30.9 * * *$ & 0.4 & 0.3 & 104 & - & - & - & 一 \\
\hline$\% \mathrm{SE}$ & $21.5 * * *$ & 0.9 & 0.7 & 104 & - & - & 一 & - \\
\hline$\%$ BE1 & $18.1 * * *$ & 1.1 & 0.9 & 104 & - & 一 & - & - \\
\hline$\%$ BE2 & 0.0 & 0.4 & 1.3 & 104 & 2.4 & 1.8 & 0.7 & 61 \\
\hline$\% \mathrm{BS}$ & $5.5^{*}$ & 0.3 & 1.8 & 102 & 一 & - & - & 一 \\
\hline $\mathrm{CS}$ & $37.5 * * *$ & $2.3^{*}$ & 1.8 & 104 & $110.9 * * *$ & 1.5 & 1.7 & 62 \\
\hline EP & 0.8 & 0.7 & 1.4 & 104 & 2.0 & 1.8 & 1.9 & 62 \\
\hline NEP & 0.0 & 1.3 & $2.3^{*}$ & 104 & 1.6 & 1.8 & 0.4 & 62 \\
\hline WL & $27.6 * * *$ & 1.3 & 0.4 & 104 & $12.9 * * *$ & $2.2 *$ & 0.2 & 62 \\
\hline SL & $1028.6 * * *$ & $6.5 * * *$ & 1.8 & 104 & $593.5^{* * *}$ & $4.3 * *$ & 0.5 & 62 \\
\hline ST & $213.9 * * *$ & $4.1 * * *$ & 1.7 & 104 & $45.9 * * *$ & $2.9 *$ & 0.7 & 62 \\
\hline $\mathbf{R t}^{\circ}$ & $26.1 * * * *$ & 0.8 & 1.1 & 104 & $19.8^{* * * *}$ & $2.4^{*}$ & 1.3 & 62 \\
\hline PUA1 & $115.9 * * *$ & 0.8 & 1.4 & 103 & $74.7 * * *$ & 0.6 & 1.5 & 62 \\
\hline PG & 0.9 & 1.7 & 2.1 & 103 & 3.9 & $2.3 *$ & 0.3 & 62 \\
\hline PUA2 & - & - & - & - & $12.5^{* * *}$ & $7.6 * * *$ & 2.1 & 62 \\
\hline WI & 一 & 一 & - & - & $14.2 * * *$ & 1.5 & 0.5 & 61 \\
\hline DMF & - & - & - & - & $50.7 * * *$ & 1.2 & 1.3 & 60 \\
\hline$\% \mathrm{MF}$ & - & - & 一 & - & $7.9 * *$ & 1.8 & $2.7^{*}$ & 60 \\
\hline
\end{tabular}

$(*),(* *),\left({ }^{* *}\right)=$ significant at 5,1 and 0.1 p. 100 level respectively. 


\section{Discussion}

We shall mainly discuss the effects associated with the dw gene, and the phenotypic correlations between traits within genotypes, separately within each experimental group. Effect of treatment per se and response of genotype to treatment will be commented with some caution, especially for traits correlated with sexual maturity, owing to the afore-mentioned higher mortality and large delay in onset of laying in heated group.

\section{A. Means and analysis of variance}

\section{a) Body weight, body weight variation food consumption and efficiency}

In general the reduction of the adult body weight in dwarfs has been reported to be around 30 p. 100 in females (HutT, 1959 ; Guillaume, 1969; Bernier \& ArscotT, 1972; Horst \& Petersen, 1978). At a given age, the larger the adult size of the strain where $\mathrm{dw}$ gene is introduced, the smaller the relative reduction of body weight according to Mohammadian \& JaAP (1972), Zloshevskaya \& PenionzhKEVICH (1972), REDDY et al. (1974). The present report seems consistent with this opinion, as the average adult body weight of the population was low and body weight reduction in dwarfs was high. The average mature and 18 -week body weight reductions in dwarfs as compared to normals were 38.4 and 36.5 p. 100 in control and 32.0 and 33.0 p. 100 in heated group respectively. On the other hand, these figures suggest that body weight reduction due to the $\mathrm{dw}$ gene is relatively less at higher temperature which seems to be is in agreement with the finding of MERAT et al. (1974), Horst \& Petersen (1978).

Body weight change may have been influenced by onset of egg production as it was significantly superior for the Dw genotype between 18 and 27 weeks in control group where peak of egg production took place, while in the corresponding period for heated group, where onset of sexual maturity was considerably delayed, dwarfs gained as much weight as normals. In the period from 27 th to 39 th, week on the contrary, dw hens have respectively in control and heated environment a lower (not significantly) body weight gain and higher body weight loss.

The reduction in observed feed consumption in dwarfs, both in control and heated group (respectively 35.7 and 36.9 p. 100) is higher than those reported by French \& Nordskog (1973), Mérat et al. (1974), Horst \& Petersen (1978) who observed a reduction between 25 and 30 p. 100 of normals. Working with birds with higher body weight than the present experiment, ARscotT \& BERNIER (1973) found that the percentage difference in body weight between adult dwarfs and nondwarfs was nearly proportional to the difference in feed consumption and FrencH \& NoRDSKog (1973) observed that dwarf pullets weighing 25 p. 100 less than nondwarfs consumed 19 p. 100 less feed. In our case the observed feed consumption of dwarfs in control group was less reduced by about 3 p. 100 compared to their body weight reduction while in heated group feed consumption was more reduced by about 5 p. 100 compared to body weight reduction. This relatively higher reduction in feed consumption compared to body weight reduction in dwarfs in our heated 
TABLE 5

Control group : test of homogeneity of correlation coefficients between $D w$ and $d w$ genotypes (upper part) and estimates for pooled genotypes when there is no significant heterogeneity (lower part).

\begin{tabular}{|c|c|c|c|c|c|c|c|c|c|}
\hline $\begin{array}{r}\chi^{2} \\
(\mathrm{~d} . \mathrm{t} . \\
=1)\end{array}$ & W2 & $\Delta \mathrm{W}$ & E & O & $\mathbf{R}$ & W1 & ASM & EN & EW2 \\
\hline W2 (1) .... & - & 0.03 & 1.96 & 1.24 & 2.63 & 0.00 & 0.10 & 2.71 & 0.74 \\
\hline$\overline{\Delta \mathrm{W}}$. & $\begin{array}{r}0.22 \\
*\end{array}$ & - & 2.69 & 1.96 & 0.03 & 0.21 & 0.02 & 3.56 & 0.02 \\
\hline$\overline{\mathrm{E} \ldots \ldots \ldots}$ & $\begin{array}{l}0.52 \\
* * *\end{array}$ & 0.07 & - & $\begin{array}{r}7.85 \\
* *\end{array}$ & $\begin{array}{r}5.96 \\
*\end{array}$ & 2.18 & 1.03 & $\begin{array}{c}6.08 \\
*\end{array}$ & 0.13 \\
\hline$\overline{o \ldots \ldots}$ & $\begin{array}{l}0.77 \\
* * *\end{array}$ & $\begin{array}{r}0.25 \\
* *\end{array}$ & $\begin{array}{ll}0.74 & \\
& 0.90\end{array}$ & - & 1.77 & 0.47 & 0.01 & $\begin{array}{c}5.29 \\
*\end{array}$ & 1.08 \\
\hline R .... & -0.17 & -0.01 & $\begin{array}{r}-0.27 \\
0.19\end{array}$ & $\begin{array}{r}0.27 \\
* *\end{array}$ & - & 0.62 & 1.17 & 2.87 & 0.25 \\
\hline$\overline{W 1 \ldots \ldots \ldots}$ & $\begin{array}{r}0.82 \\
* * *\end{array}$ & 0.13 & $\begin{array}{r}0.41 \\
* * *\end{array}$ & $\begin{array}{r}0.63 \\
* * *\end{array}$ & -0.11 & - & 0.04 & 2.68 & 0.45 \\
\hline$\overline{\mathrm{ASM}}$ & 0.17 & -0.11 & 0.07 & 0.09 & -0.04 & 0.06 & 一 & 0.01 & 0.71 \\
\hline$\overline{\mathrm{EN}}$ & $\begin{array}{r}0.31 \\
* *\end{array}$ & $\overline{0.14}$ & $\begin{array}{ll}0.80 & \\
& 0.92 \\
\end{array}$ & $\begin{array}{ll}0.53 & \\
& 0.78 \\
\end{array}$ & -0.00 & $\begin{array}{r}0.24 \\
*\end{array}$ & $\begin{array}{r}-0.26 \\
* *\end{array}$ & - & 0.12 \\
\hline$\overline{\text { EW2 (2) } \ldots .}$ & $\begin{array}{r}0.44 \\
* * *\end{array}$ & 0.17 & $\begin{array}{l}0.46 \\
* * *\end{array}$ & $\begin{array}{l}0.46 \\
* * *\end{array}$ & -0.12 & $\begin{array}{r}0.42 \\
* * *\end{array}$ & -0.05 & 0.05 & \\
\hline$\% \mathrm{ES}$. & $\begin{array}{c}0.44 \\
0.01 \\
\end{array}$ & 0.04 & -0.11 & $\begin{array}{r}-0.32 \\
0.07\end{array}$ & $\begin{array}{r}0.19 \\
*\end{array}$ & $\begin{array}{r}-0.24 \\
* *\end{array}$ & $\begin{array}{r}-0.21 \\
0.18\end{array}$ & 0.09 & 0.03 \\
\hline$\overline{Y / A(2) \ldots .}$ & -0.06 & $-\overline{-0.09}$ & $\begin{array}{r}-0.28 \\
* *\end{array}$ & -0.19 & 0.05 & -0.17 & 0.04 & $\begin{array}{r}0.00 \\
-0.37\end{array}$ & $\begin{array}{r}0.45 \\
* * *\end{array}$ \\
\hline$\overline{\text { EST (3) } \ldots}$ & $\begin{array}{r}-0.07 \\
0.35\end{array}$ & 0.10 & $\begin{array}{r}-0.14 \\
0.60\end{array}$ & $\begin{array}{r}-0.17 \\
0.55\end{array}$ & $\begin{array}{r}-0.11 \\
0.28\end{array}$ & $\begin{array}{r}-0.10 \\
0.40\end{array}$ & 0.06 & $\begin{array}{r}-0.24 \\
0.42\end{array}$ & 0.13 \\
\hline$\%$ DYE $\ldots$. & 0.01 & -0.05 & -0.04 & 0.03 & 0.10 & 0.14 & -0.05 & -0.07 & 0.11 \\
\hline$\% \mathrm{SE} \ldots \ldots$ & -0.05 & 0.03 & -0.07 & -0.11 & -0.13 & -0.08 & -0.07 & -0.12 & $\stackrel{0.21}{-0.21}$ \\
\hline$\% \mathrm{BE} 2$ & $\begin{array}{r}0.19 \\
*\end{array}$ & 0.16 & 0.04 & 0.18 & 0.07 & 0.17 & 0.06 & 0.00 & 0.15 \\
\hline$\overline{C S} \ldots$ & $\begin{array}{ll}0.14 & \\
& 0.52 \\
\end{array}$ & 0.05 & $\begin{array}{r}0.50 \\
* * *\end{array}$ & $\begin{array}{ll}0.30 & \\
& 0.60 \\
\end{array}$ & 0.01 & $\begin{array}{r}0.06 \\
0.50\end{array}$ & $\begin{array}{c}0.20 \\
*\end{array}$ & $\begin{array}{l}0.46 \\
* * *\end{array}$ & -0.10 \\
\hline$\overline{\mathrm{EP}}$ & $\begin{array}{r}0.35 \\
* * * \\
\end{array}$ & -0.04 & $\begin{array}{l}-0.87 \\
-0.96 \\
\end{array}$ & $\begin{array}{c}0.49 \\
-0.82 \\
\end{array}$ & $\begin{array}{l}0.36 \\
-0.20\end{array}$ & $\begin{array}{r}-0.21 \\
*\end{array}$ & -0.03 & $\begin{array}{r}-0.77 \\
-0.33\end{array}$ & $\begin{array}{r}-0.27 \\
* * \\
\end{array}$ \\
\hline$\overline{\mathrm{Rt}^{\circ}}$ & -0.15 & -0.00 & $\begin{array}{r}-0.38 \\
* * * \\
\end{array}$ & $-0.22 *$ & 0.16 & $\begin{array}{r}-0.22 \\
*\end{array}$ & -0.01 & $\begin{array}{r}0.37 \\
* * *\end{array}$ & -0.07 \\
\hline PUA1. & -0.02 & 0.01 & -0.18 & -0.08 & 0.13 & -0.17 & -0.01 & $\begin{array}{r}0.39 \\
-0.03\end{array}$ & 0.08 \\
\hline$\overline{\mathrm{PG}}$ & -0.08 & -0.07 & -0.10 & -0.14 & -0.08 & -0.02 & -0.16 & 0.03 & 一 0.10 \\
\hline
\end{tabular}

(1) d.f. $=114$ for all traits except those indicated (2) or (3).

(2) d.f. $=101$.

(3) d.f. $=105$.

$(*),(* *),(* * *)=$ Significantly different from zero respectively at 5,1 and 0.1 p. 100 level. 
Lot témoin : test d'homogénéité des coefficients de corrélation entre les génotypes $D w$ et $d w$ (partie supérieure) et estimations pour les génotypes groupés lorsqu'il n'y a pas d'hétérogénéité significative (partie inférieure).

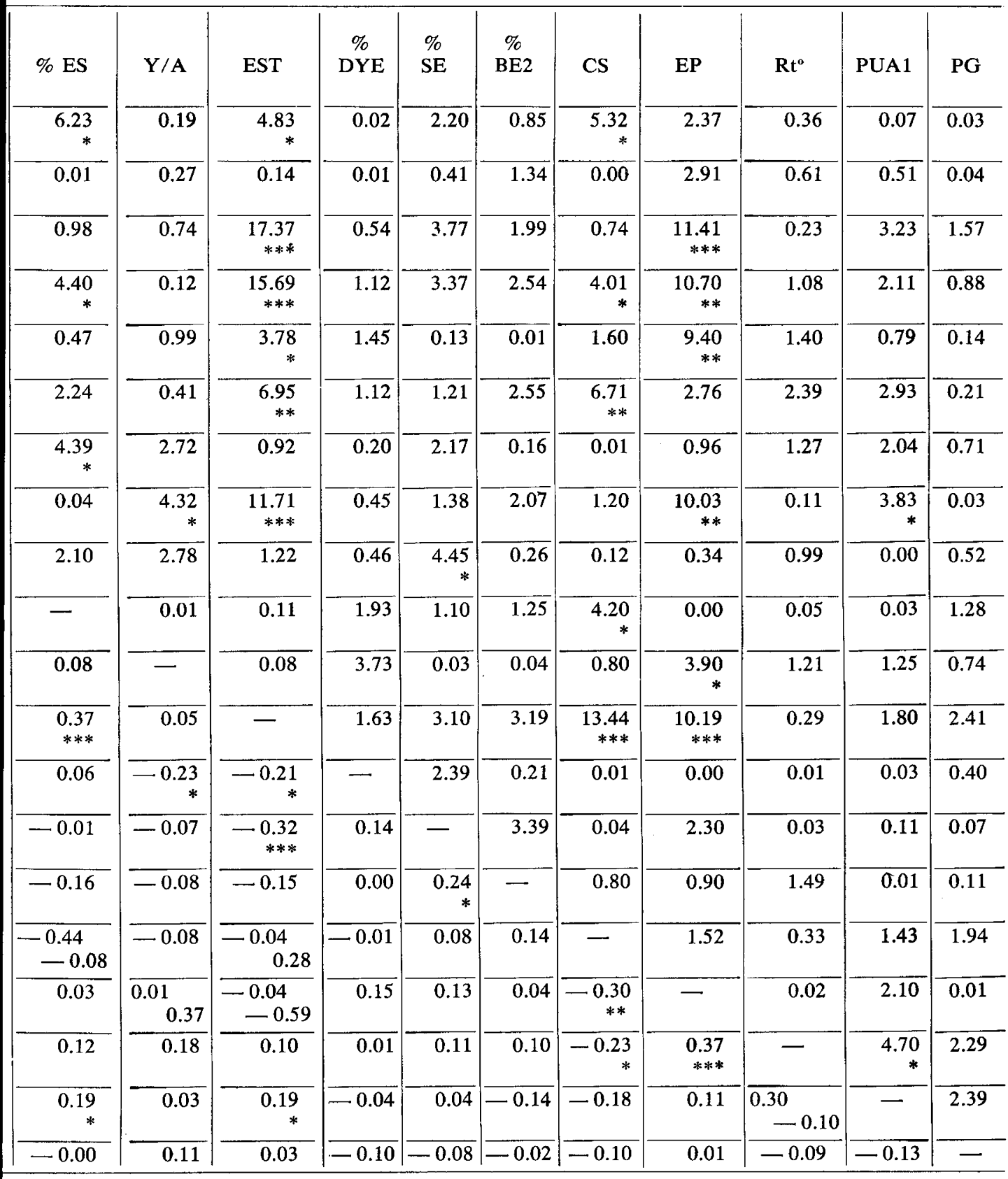

In the lower part, in cases where there is significant heterogeneity between genotypes as shown by the upper part, the values of the correlation coefficient are given respectively for $\mathrm{Dw}$ and $\mathrm{dw}$, instead of a single value. 


\section{TABLE 6}

Heated group : test of homogeneity of correlation coefficients between $D w$ and $d w$ genotypes (upper part) and estimates for pooled genotypes when there is no significant heterogeneity.

Lot chauffé : test d'homogénéité des coefficients de corrélation entre les génotypes $D w$ et $d w$ (partie supérieure) et estimations pour les génotypes groupés lorsqu'il n'y a pas d'hétérogénéité significative (partie inférieure).

\begin{tabular}{|c|c|c|c|c|c|c|c|}
\hline $\begin{array}{r}\chi^{2} \\
\text { (d.f. } \\
=1)\end{array}$ & W2 & $\Delta W$ & E & $\mathrm{O}$ & $\mathbf{R}$ & W1 & ASM \\
\hline$\overline{\mathrm{W} 2(1) \ldots \ldots \ldots}$ & - & $\begin{array}{r}9.08 \\
* *\end{array}$ & $\begin{array}{r}4.33 \\
*\end{array}$ & $\begin{array}{r}5.57 \\
*\end{array}$ & 0.16 & $\begin{array}{r}4.28 \\
*\end{array}$ & $\begin{array}{r}7.77 \\
* * \\
\end{array}$ \\
\hline$\overline{\Delta \mathrm{W}}$. & $\begin{array}{l}0.55 \\
-0.12\end{array}$ & - & $\begin{array}{r}4.32 \\
*\end{array}$ & 1.24 & $\begin{array}{r}11.18 \\
* * *\end{array}$ & 1.08 & 0.33 \\
\hline$\overline{\mathrm{E}}$ & $\begin{array}{ll}0.43 & \\
& 0.29\end{array}$ & $\begin{array}{ll}0.50 & \\
& 0.05\end{array}$ & - & 0.75 & 1.61 & 0.80 & 0.00 \\
\hline$\overline{\mathrm{O}}$ & $\begin{array}{ll}0.77 & \\
& 0.43 \\
\end{array}$ & $\begin{array}{l}0.46 \\
* * *\end{array}$ & $\begin{array}{c}0.86 \\
* * *\end{array}$ & - & 1.60 & 0.37 & 1.01 \\
\hline$\overline{\mathbf{R} \ldots \ldots}$ & $\begin{array}{r}0.39 \\
* * *\end{array}$ & $\begin{array}{r}-0.40 \\
0.36\end{array}$ & $\begin{array}{r}-0.26 \\
*\end{array}$ & 0.09 & - & 0.58 & 0.17 \\
\hline$\overline{W 1}$ & \begin{tabular}{|ll}
0.85 & \\
& 0.64 \\
\end{tabular} & $\begin{array}{r}0.30 \\
* *\end{array}$ & $\begin{array}{l}0.55 \\
* * *\end{array}$ & $\begin{array}{r}0.63 \\
* * * \\
\end{array}$ & $\begin{array}{r}-0.33 \\
* *\end{array}$ & - & 0.58 \\
\hline$\overline{\text { ASM . . }}$ & $\begin{array}{r}-0.21 \\
0.43 \\
\end{array}$ & -0.19 & $\begin{array}{r}-0.37 \\
* *\end{array}$ & $\begin{array}{r}-0.35 \\
* *\end{array}$ & -0.21 & -0.14 & - \\
\hline$\overline{\mathrm{EN}}$. & $\begin{array}{c}0.42 \\
-0.21\end{array}$ & $\begin{array}{r}0.30 \\
* *\end{array}$ & $\begin{array}{l}0.67 \\
* * *\end{array}$ & $\begin{array}{r}0.63 \\
* * *\end{array}$ & 0.08 & $\begin{array}{r}0.32 \\
* *\end{array}$ & $\begin{array}{r}0.86 \\
* * *\end{array}$ \\
\hline EW2 . & $\begin{array}{r}0.52 \\
* * * \\
\end{array}$ & $\stackrel{0.42}{-0.22}$ & $\begin{array}{l}0.61 \\
* * *\end{array}$ & $\begin{array}{l}0.55 \\
* * *\end{array}$ & $\begin{array}{c}0.56 \\
-0.08\end{array}$ & $\begin{array}{r}0.53 \\
* * *\end{array}$ & -0.03 \\
\hline$\overline{\% \text { ES (2) } \ldots \ldots}$ & $\begin{array}{r}-0.19 \\
0.29 \\
\end{array}$ & 0.15 & \begin{tabular}{|ll}
0.09 & \\
& 0.65 \\
\end{tabular} & $\begin{array}{r}0.04 \\
0.64 \\
\end{array}$ & 0.00 & 0.10 & -0.15 \\
\hline$\overline{Y / A(2) \ldots \ldots .}$ & 0.22 & 0.02 & 0.00 & 0.17 & 0.17 & 0.08 & -0.07 \\
\hline$\overline{\text { EST (2) } \ldots \ldots \ldots}$ & 0.05 & $\begin{array}{r}-0.19 \\
0.36\end{array}$ & $\begin{array}{r}-0.05 \\
0.59 \\
\end{array}$ & $\begin{array}{r}-0.18 \\
0.57\end{array}$ & -0.12 & $\begin{array}{r}0.23 \\
*\end{array}$ & 0.13 \\
\hline$\%$ BE2 $\ldots \ldots \ldots$ & 0.01 & 0.03 & 0.00 & -0.05 & -0.14 & -0.10 & -0.03 \\
\hline$\overline{\mathrm{CS}} \ldots$ & $\begin{array}{l}0.49 \\
* * * \\
\end{array}$ & $\begin{array}{r}0.26 \\
* \\
\end{array}$ & $\begin{array}{r}0.71 \\
* * * \\
\end{array}$ & $\begin{array}{r}0.72 \\
* * * \\
\end{array}$ & -0.09 & $\begin{array}{r}0.44 \\
* * * \\
\end{array}$ & $\begin{array}{r}-0.33 \\
* * \\
\end{array}$ \\
\hline$\overline{\mathrm{EP}} \ldots$ & -0.21 & $\begin{array}{r}-0.26 \\
*\end{array}$ & $\begin{array}{r}-0.56 \\
* * *\end{array}$ & $\begin{array}{r}0.46 \\
* * * \\
\end{array}$ & 0.16 & -0.17 & $\begin{array}{r}0.19 \\
\quad-0.30\end{array}$ \\
\hline $\mathrm{Rt}^{\mathrm{o}} \ldots \ldots \ldots \ldots$ & 0.00 & 0.10 & -0.02 & 0.02 & 0.04 & -0.05 & -0.14 \\
\hline PUA1 $\ldots \ldots \ldots$ & 0.04 & 0.16 & -0.12 & -0.05 & 0.00 & -0.07 & 0.16 \\
\hline PG $\ldots \ldots \ldots \ldots$ & 0.17 & -0.02 & -0.07 & 0.03 & 0.02 & 0.16 & 0.02 \\
\hline$\overline{\text { PUA2 } \ldots \ldots \ldots}$ & $\begin{array}{r}-0.28 \\
* \\
\end{array}$ & $\begin{array}{r}0.57 \\
* * *\end{array}$ & $\begin{array}{r}-0.25 \\
* \\
\end{array}$ & $\begin{array}{r}-0.29 \\
* \\
\end{array}$ & 0.23 & $\begin{array}{r}0.27 \\
*\end{array}$ & 0.05 \\
\hline WI . . & 0.22 & -0.01 & 0.18 & 0.19 & $\longrightarrow 0.09$ & $\begin{array}{r}0.36 \\
* * \\
\end{array}$ & 0.07 \\
\hline$\overline{\mathrm{DMF}}$ & $\begin{array}{r}0.29 \\
*\end{array}$ & 0.21 & $\begin{array}{l}0.48 \\
* * *\end{array}$ & $\begin{array}{l}0.59 \\
* * *\end{array}$ & 0.16 & $\begin{array}{r}0.32 \\
* * \\
\end{array}$ & -0.14 \\
\hline$\%$ DMF & $\begin{array}{r}0.37 \\
* *\end{array}$ & 0.03 & 0.16 & $\begin{array}{r}0.32 \\
* *\end{array}$ & 0.09 & 0.11 & 0.09 \\
\hline
\end{tabular}

(1) d.f. $=73$.

(2) d.f. $=70$.

$(*),(* *),(* * *)=$ significantly different from zero respectively at 5,1 and 0.1 p. 100 level.

In the lower part, in cases where there is significant heterogeneity between genotypes as shown by the upper part, the values of the correlation coefficient are given within parentheses respectively for $\mathrm{Dw}$ and $\mathrm{dw}$, instead of a single value. 
TABLE 6 (continued)

\begin{tabular}{|c|c|c|c|c|c|c|c|}
\hline$r \quad \begin{array}{r}x^{2} \\
(\mathrm{~d} . \mathrm{f} . \\
=1)\end{array}$ & EN & EW2 & $\% \mathrm{ES}$ & $\mathbf{Y} / \mathbf{A}$ & EST & $\% \mathrm{BE} 2$ & $\mathrm{CS}$ \\
\hline$\overline{W 2(1) \ldots \ldots \ldots}$ & $\begin{array}{r}7.42 \\
* *\end{array}$ & 0.10 & $\begin{array}{r}3.84 \\
*\end{array}$ & 0.22 & 1.96 & 0.33 & 1.21 \\
\hline$\overline{\Delta W \quad \ldots \ldots \ldots}$ & 0.81 & $\begin{array}{r}7.85 \\
* *\end{array}$ & 2.67 & 1.59 & $\begin{array}{r}5.24 \\
*\end{array}$ & 0.84 & 0.38 \\
\hline $\bar{E} \ldots$ & 0.11 & 0.32 & $\begin{array}{r}6.12 \\
*\end{array}$ & 2.29 & $\begin{array}{r}8.66 \\
* *\end{array}$ & 2.90 & 0.16 \\
\hline$O \ldots \ldots$ & 1.44 & 2.27 & $\begin{array}{r}9.99 \\
* *\end{array}$ & 1.91 & $\begin{array}{r}11.30 \\
* * *\end{array}$ & 0.65 & 0.74 \\
\hline $\mathbf{R} \ldots \ldots \ldots \ldots$ & 0.30 & $\begin{array}{r}5.17 \\
*\end{array}$ & 0.56 & 0.19 & 0.08 & 1.43 & 0.01 \\
\hline$\overline{\mathrm{W} 1} \ldots \ldots \ldots$ & 0.48 & 0.48 & 2.14 & 0.00 & 3.80 & 0.14 & 0.37 \\
\hline$\overline{\operatorname{ASM} \ldots \ldots \ldots}$ & 2.54 & 1.00 & 0.50 & 0.69 & 0.48 & 0.07 & 1.05 \\
\hline$\overline{\mathrm{EN}} \ldots \ldots$ & 一 & 0.33 & 1.87 & 0.00 & 3.64 & 0.90 & 0.13 \\
\hline$\overline{\text { EW2 }}$ & 0.15 & - & 3.53 & $\begin{array}{r}4.94 \\
*\end{array}$ & 0.62 & 0.11 & 1.05 \\
\hline$\overline{\% \text { ES (2) } \ldots \ldots}$ & $\begin{array}{r}0.29 \\
*\end{array}$ & -0.10 & - & $\begin{array}{r}8.42 \\
* *\end{array}$ & 1.48 & 0.00 & $\begin{array}{r}4.55 \\
*\end{array}$ \\
\hline$\overline{Y / A ~(2) ~}$ & 0.16 & $\begin{array}{r}0.41 \\
0.11\end{array}$ & $\begin{array}{r}0.03 \\
0.60\end{array}$ & - & 0.61 & 0.31 & 0.72 \\
\hline EST (2) & 0.00 & 0.13 & $\begin{array}{r}0.51 \\
* * *\end{array}$ & 0.17 & 一 & 0.02 & $\begin{array}{r}5.11 \\
*\end{array}$ \\
\hline$\%$ BE2 $\ldots \ldots \ldots$ & 0.06 & -0.04 & -0.05 & 0.10 & -0.06 & - & 2.97 \\
\hline$\overline{\mathrm{CS}}$ & $\begin{array}{r}0.66 \\
* * *\end{array}$ & $\begin{array}{r}0.25 \\
*\end{array}$ & $\begin{array}{ll}0.00 & \\
& 0.49\end{array}$ & 0.16 & $\begin{array}{c}0.16 \\
0.37\end{array}$ & 0.13 & - \\
\hline EP $\ldots$ & $\begin{array}{r}-0.42 \\
* * *\end{array}$ & -0.12 & $\begin{array}{r}-0.30 \\
* *\end{array}$ & -0.13 & $\stackrel{0.08}{-0.42}$ & 0.01 & $\begin{array}{r}-0.41 \\
* * *\end{array}$ \\
\hline$\overline{\mathbf{R t}^{\mathbf{o}}} \ldots$ & 0.13 & -0.12 & 0.21 & 0.04 & -0.00 & 0.06 & 0.00 \\
\hline PUA1. & -0.20 & $\begin{array}{l}0.34 \\
-0.25 \\
\end{array}$ & -0.13 & 0.07 & -0.16 & -0.05 & $\begin{array}{r}-0.23 \\
*\end{array}$ \\
\hline$\overline{\mathrm{PG}}$ & -0.02 & 0.03 & 0.02 & $\begin{array}{l}0.37 \\
\quad 0.10\end{array}$ & 0.16 & 0.14 & 0.03 \\
\hline$\overline{\text { PUA2 } \ldots \ldots \ldots}$ & -0.15 & 0.00 & $\begin{array}{r}-0.36 \\
* *\end{array}$ & 0.01 & $\stackrel{0.11}{-0.55}$ & -0.04 & -0.19 \\
\hline$\overline{\text { WI . . }}$ & -0.00 & $\begin{array}{r}0.19 \\
0.33 \\
\end{array}$ & 0.26 & 0.06 & 0.22 & -0.06 & 0.20 \\
\hline DMF . & $\begin{array}{r}0.30 \\
* * \\
\end{array}$ & $\begin{array}{r}0.06 \\
0.44 \\
\end{array}$ & 0.05 & 0.13 & $\begin{array}{c}-0.04 \\
-0.42 \\
\end{array}$ & -0.02 & $\begin{array}{r}0.36 \\
* * \\
\end{array}$ \\
\hline$\overline{\% \text { DMF } \ldots}$ & 0.01 & $\begin{array}{r}0.26 \\
*\end{array}$ & -0.15 & 0.15 & 0.17 & 0.08 & 0.07 \\
\hline
\end{tabular}

(1) d.f. $=73$.

(2) d.f. $=70$

$\left({ }^{*}\right),(* *),(* * *)=$ significantly different from zero respectively at 5,1 and 0.1 p. 100 level.

In the lower part, in cases where there is significant heterogeneity between genotypes as shown by the upper part, the values of the correlation coefficient are given within parentheses respectively for Dw and dw, instead of a single value. 
TABLE 6 (continued)

\begin{tabular}{|c|c|c|c|c|c|c|c|c|}
\hline $\begin{array}{r}\chi^{2} \\
\text { (d.f. } \\
=1)\end{array}$ & EP & $\mathbf{R t}^{\circ}$ & PUA1 & PG & PUA2 & WI & $\mathrm{DMF}$ & $\%$ DMF \\
\hline W2 (1) $\ldots$ & 1.26 & 0.40 & 1.92 & 1.12 & 0.01 & 0.15 & 0.01 & 0.16 \\
\hline$\overline{\Delta W} \quad \ldots \ldots$ & 0.92 & 0.58 & $0 . \overline{04}$ & 0.75 & 0.93 & 0.88 & 0.01 & 1.60 \\
\hline $\mathrm{E} \ldots \ldots \ldots$ & 3.31 & 0.16 & 1.46 & 2.22 & 0.06 & 2.14 & 0.62 & 0.91 \\
\hline $\mathbf{O} \ldots \ldots$ & 1.88 & 0.05 & 0.48 & 1.20 & 0.08 & 2.26 & 1.46 & 0.36 \\
\hline $\mathbf{R} \ldots$ & 0.58 & 2.51 & 3.05 & 2.07 & 0.56 & 0.00 & 0.30 & 0.00 \\
\hline W1 . & 0.83 & 1.72 & 2.81 & 1.96 & 0.44 & 0.01 & 0.26 & 0.44 \\
\hline$\overline{\mathrm{ASM}}$ & $\begin{array}{r}4.26 \\
*\end{array}$ & 1.25 & 0.91 & 0.24 & 2.03 & 1.07 & 1.21 & 0.29 \\
\hline$\overline{\mathrm{EN}} \ldots$ & 1.31 & 0.79 & 0.00 & 0.99 & 0.64 & 0.15 & 0.63 & 1.11 \\
\hline EW2 & 1.87 & 0.77 & $\begin{array}{r}6.25 \\
*\end{array}$ & 3.46 & 0.15 & $\begin{array}{r}4.61 \\
*\end{array}$ & $\begin{array}{r}4.56 \\
*\end{array}$ & 0.22 \\
\hline$\% \operatorname{ES~(2)~}$ & 2.52 & 0.00 & 1.90 & 0.16 & 0.13 & 0.01 & 0.81 & 3.17 \\
\hline Y/A (2) & 0.30 & 1.01 & 1.01 & $\begin{array}{r}3.87 \\
*\end{array}$ & 1.15 & 0.43 & 0.13 & 0.02 \\
\hline EST (2) & $\begin{array}{r}4.59 \\
*\end{array}$ & 1.66 & 0.26 & 0.01 & $\begin{array}{r}9.00 \\
* *\end{array}$ & 1.94 & $\begin{array}{r}3.95 \\
*\end{array}$ & 0.03 \\
\hline$\%$ BE2 $\ldots \ldots$ & 0.08 & 0.10 & 2.13 & 0.89 & 0.10 & 0.01 & 0.22 & 0.51 \\
\hline CS $\ldots$ & 0.00 & 0.02 & 0.27 & 0.84 & 0.27 & 0.01 & 1.52 & 0.02 \\
\hline $\mathrm{EP} \ldots$ & - & 0.65 & 2.18 & 0.09 & 0.06 & $\begin{array}{r}8.58 \\
* * \\
\end{array}$ & 0.61 & 1.97 \\
\hline $\mathrm{Rt}^{\circ}$ & -0.10 & 一 & 2.23 & 0.88 & 1.78 & 0.91 & 0.01 & 2.63 \\
\hline$\overline{\text { PUA } 1 .}$ & 0.00 & 0.07 & - & $\begin{array}{r}3.84 \\
*\end{array}$ & 0.01 & 2.92 & 0.40 & 0.87 \\
\hline$\overline{\mathrm{PG}}$ & 0.05 & -0.07 & $\begin{array}{l}0.12 \\
-0.34\end{array}$ & - & 3.25 & 1.15 & 1.17 & 0.32 \\
\hline PUA2 & 0.19 & $\begin{array}{r}-0.41 \\
* * *\end{array}$ & 0.04 & 0.17 & - & 0.58 & 0.11 & 1.15 \\
\hline WI & $\begin{array}{l}0.34 \\
-0.35 \\
\end{array}$ & 0.15 & -0.13 & -0.01 & -0.23 & - & 0.78 & 0.86 \\
\hline$\overline{\mathrm{DMF}}$ & $\begin{array}{r}-0.24 \\
*\end{array}$ & -0.15 & 0.00 & 0.06 & -0.19 & 0.10 & - & 2.07 \\
\hline$\% \mathrm{DMF}$ & -0.12 & -0.24 & 0.06 & -0.06 & 0.11 & $\begin{array}{r}0.49 \\
* * * \\
\end{array}$ & 0.16 & - \\
\hline
\end{tabular}

(1) d.f. $=73$.

(2) d.f. $=70$.

$(*),(* *),(* * *)=$ significantly different from zero respectively at 5,1 and 0.1 p. 100 level.

In the lower part, in cases where there is significant heterogeneity between genotypes as shown by the upper part, the values of the correlation coefficient are given within parentheses respectively for $D w$ and $d w$, instead of a single value. 


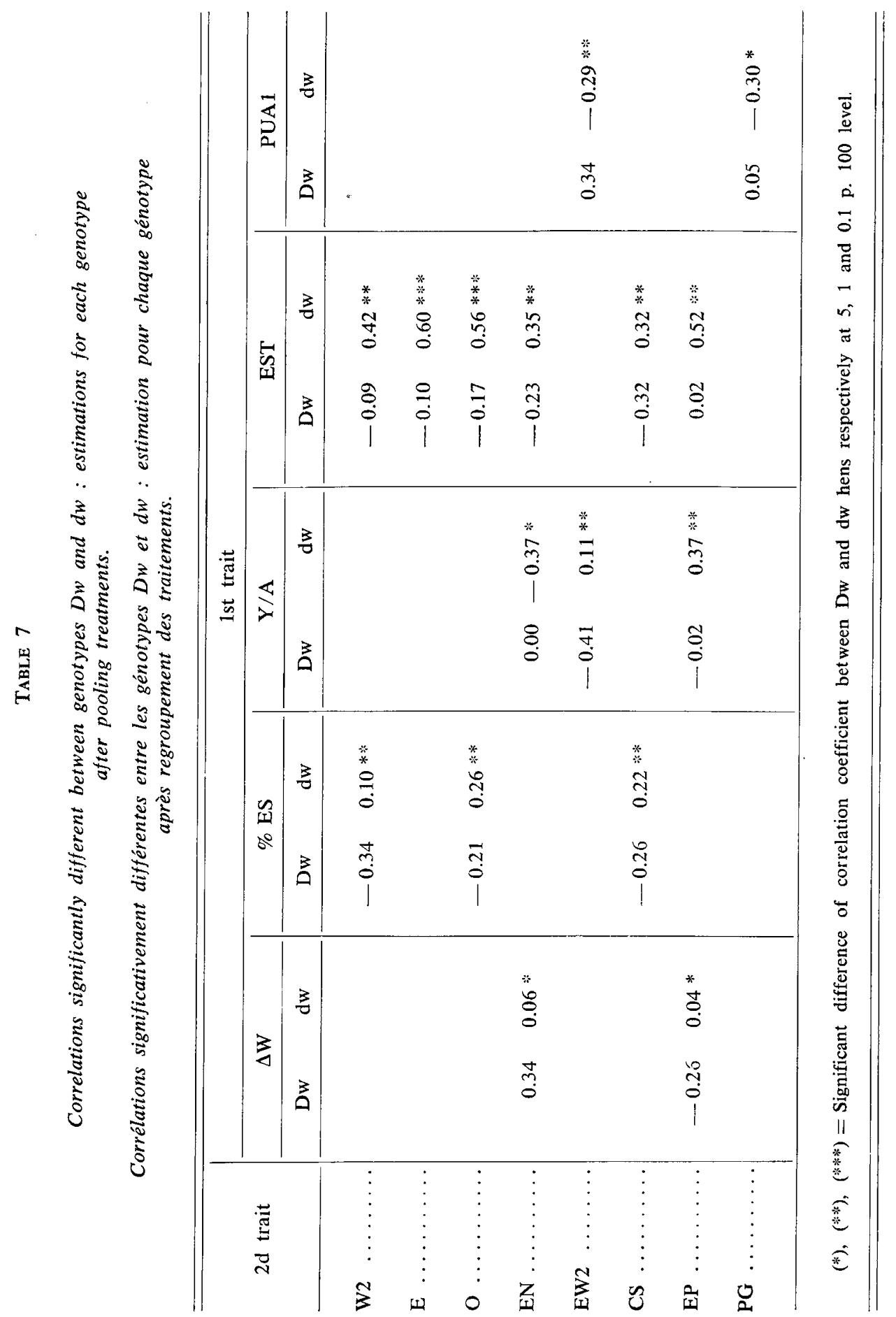


group seems mainly to be due to the considerably lower egg production in dwarfs compared to normal in this group. In a previous experiment (Mérat et al., 1974) heat cauded a relatively higher reduction in food consumption in normales compared to dwarfs.

The significantly lower $R$ (residual food consumption) at 5 p. 100 level in dwarfs in heated group (joined to the fact that in both groups $R$ was positive in Dw and negative in dw genotype), suggests a better feed efficiency of dwarfs at higher temperature and possibly also at moderate temperature. This was not found in the report of Prod'homme \& MÉrat (1969) in a population of larger body size. The highly significant sire family effect for residual feed consumption in control and in heated group, observed in our study, has also been reported by Bordas \& MÉrAT (1981). This sire family effect on $R$ may suggest a possible improvement of this trait through sire family selection. On the other hand, it must be mentioned that $r^{2}$ (the square of the correlation coefficient between observed and estimated individual food consumption) in our study was in the order 95-96 p. 100 as also observed by NordSKoG (1973) in white Leghorn. In brown-egg stock MÉrat et al. (1974, 1980), Bordas \& MÉrat $(1976,1981)$ reported this value to be around $80 \mathrm{p} .100$. Thus it seems that in Leghorns scope of improving the residual consumption is considerably less than in other breeds.

Feed efficiency, in terms of feed consumed per unit of egg mass produced, was 2.7 for dwarfs compared to 2.8 for normal in control group while it was 2.5 in heated group for both genotypes. Thus in our case, heat appeared to improve feed efficiency to the same level in both genotypes. Mérat et al. (1974) found a slightly better feed efficiency for dwarfs at high temperature and HoRst \& PETERSEN (1978) working with higher body weight laying hens observed a better feed efficiency for dwarfs while in a lower body weight group, a lower feed efficiency for dwarfs compared to normals was found in a heated group. It appears that dwarf gene has a beneficial effect on feed efficiency mainly when introduced in a line with not too low body weight.

\section{b) Egg production traits}

Reduction in egg number from 1st egg to 39 weeks of age in dwarfs was 20.2 and 40.9 p. 100 respectively in control and heated group. Delay in age at sexual maturity in dwarfs was only one day in control while in heated group it was 19 days on average. Clutch size was significantly lower in dwarfs than in normals in both control and heated group but the reduction in control group was particularly high. Laying pause days and number of pauses were higher in dwarfs but not significantly different from Dw genotype. The consequence of this was reduced laying rate. Corresponding to this and to lower mean egg weight, egg mass per 28 days $(E)$ in dwarf hens, is reduced by 33.8 and 37.5 p. 100 respectively in control and heated environment as compared to normals.

Previous estimates of reduction of egg production in dwarfs have not been consistent ranging from zero to 36 p. 100 . A reduction in laying rate of 10 p. 100 or more has been reported with medium or light stocks (HUTT, 1959; BERNIER \& Arscott, 1960; MÉrat, 1969 ; Polkinghorne \& Lowe, 1973 ; Quisenberry \& Bradley, 1971 ; Quisenberry, 1972 ; Summers, 1973 ; Doran \& Quisenberry, 
1974). The present higher reduction in control group may be mainly due to lower adult body weight of the population. This is in line with observation of a positive correlation between egg production and body weight among dw hens of small to moderate size (MéRAT, 1969). This reduction in control group is little less than observed by Horst \& Petersen (1978) (22.3 p. 100) in a population of dwarf White Leghorn. The total egg mass reduction in dwarfs is also higher than reported in the literature (21-28 p. 100). This higher reduction may be expected because of the higher body weight reduction and consequently egg weight reduction in dwarfs. Age at sexual maturity is generally delayed by 7-10 days in dwarfs (HUTT, 1959; MÉrat, 1969 ; Bernier \& ARscotT, 1960 ; Ricard \& Cochez, 1972). Horst \& Petersen (1978) reported this delay to be 9-15 days while Leclerce \& Blum (1975) and Guillaume (1975) observed a significant opposite difference. So in the present report the delay of one day in control group is less than generally found before. Concerning clutch size, Mérat (1972) reported a lower reduction of it in dwarfs compared to the present experiment, but body weights were higher in the former study. Small clutch size in dwarfs may be attributed to less active vitellogenesis (JaAP \& MoHammadran, 1969). On the other hand, delayed ovulation of a hormonal or nutritional nature corresponding to short term calcium need cannot be ruled out as dwarfs appear to consume significantly more food than normals during the days of egg formation (BoRdas \& MÉrAT, 1976).

As mentioned before, in the heated group some factors other than temperature, possibly associated with Marek's disease contamination, are likely to have interfered with egg production traits, mainly by delaying sexual maturity. This may explain why reduction in egg production in heated group was higher than reported by various authors. On the other hand, laying rate during the control period is not so much reduced. This comes from the fact that although clutch size is obviously less in the group at higher temperature, this is partly compensated by the lower mean number of pause days. Finally, egg mass per 28 days is not much lower in heated compared to control group, which partly corresponds to the limited reduction of body and mean egg size by the former environment.

\section{c) Egg traits}

Reduction in egg weight in dwarfs was 14.2 and 10.7 p. 100 , when measured during the first and last 15 days of test period (27-39 weeks) respectively in control group. In heated group this reduction was about 1 p. 100 more during the last 15 days of the test period. A highly significant sire family effect and a significant genotype $\times$ family interaction effect was observed in egg weight measured during the first 15 days of test period, suggesting the existence of genetic modifiers of the effects of the $d w$ gene on this trait. In comparison, the ratio of mean egg weight of $\mathrm{dw}$ hens compared to normals has generally been estimated around 0.90 or more. Bernier \& ARSCOTt (1972) obtained the ratio 0.95 while Dorminey et al. (1973) found its value to be 0.97. Leclerco \& Blum (1979) and Guillaume (1975) suggested that there is a constant difference in absolute egg weight between dwarf and normal hens instead of a constant ratio. On the other hand, Yamada et al. (1972) observed the increase of this ratio from 0.95 to 0.98 from 150 to 350 days of age while in the present report it increased from 0.85 to 0.89 from 27 to 39 weeks of age. 
Lillie et al. (1976), Carmon \& Huston (1976) and de Andrade et al. (1976) observed 8.5 to 10 p. 100 reduction in egg weight due to heat. The absence of a similar detectable effect in our data may be attributed to abnormally higher age at sexual maturity and relatively lower reduction in adult body weight in our heated group.

The reduction in albumen and yolk weight in dwarfs as compared to normals in the present experiment was the same (14 p. 100) in control group. In heated group the corresponding reduction in yolk weight was higher compared to albumen (20.5 v 11 p. 100) hence yolk/albumen ratio is significantly lower for dw hens in heated group. A highly significant genotype $\times$ family interaction was observed for yolk/ albumen ratio in heated group. However, concerning the egg components, albumen and yolk, caution is necessary in interpreting effects of genotype according to treatment, as the measurement was done at a later age in heated group. For albumen height, it was reduced significantly at 5 p. 100 level in dwarfs in control group, while in heated group a highly significant increase was observed for this genotype. Sire family interaction effect for albumen height was significant at 5 p. 100 level only in control group.

Previously MÉrat (1970, 1972) compared egg traits of dwarf and normal hens and reported no difference in yolk/albumen ratio as observed here in control group.

A reduction in egg shell thickness was present for dwarfs, relatively higher in heated group, and the difference between genotypes was very highly significant in this group and at 5 p. 100 level in control group. While sire family effect was significant at 5 percent in both control and heated group, interaction was only significant in the latter group. There was no significant difference between genotypes for percent egg shell but in heated group dwarfs have lower and in control group higher percent egg shell compared to normals. Sire family effect was highly significant only in control group. Percent double yolks, soft and broken eggs during the first 7 weeks of laying were very highly significantly lower and percent blood spots was lower at 5 p. 100 level in dwarfs in control group. In heated group it is recalled that these traits were not measured due to the delay in sexual maturity. Percent broken eggs in dwarfs from 27th to 30th week was higher in heated and lower in control group compared to normals, though not significantly different.

In previous experiments, a lower egg density in Leghorn dwarf hens was reported by BERNIER \& ARSCOTt (1960) and in an experimental cross by Gleichauf (1974). While these reports correspond with the present one, MÉrat (1972) found no difference in egg shell thickness in dwarf and normal medium size hens. Fewer broken eggs in dwarfs reported by Ricard (1972), RAPP (1972) and Silber \& MÉRAT (1974) are consistent with the present report as concerns control group. Reduction of double yolked and soft shelled eggs in the present paper agrees with the results of VAN MiddelKoop (1973) and Silber \& Mérat (1974).

Reduction of egg shell thickness in dwarfs especially in the heated group appears at least partly due to insufficient availability of calcium from feed due to lower consumption and from body due to lower body weight in dwarfs. HorsT \& Petersen (1978) observed a lower shell quality and thickness of eggs in dwarfs maintained under higher temperature compared to normal temperature. 


\section{d) Anatomical and physiological traits}

On the whole, rectal temperature was $0.1^{\circ} \mathrm{C}$ higher in heated group. Whilc effect of genotype was highly significant in both groups, sire family effect was significant at 5 p. 100 level only in heated group. The reduced body temperature in dwarfs may be due to their lower metabolic rate caused by lower thyroid activity (Mérat \& Guillaume, 1969, and others cited by Guillaume, 1976).

As has been already reported (BANERJEE et al., 1981) a highly significant increase in plasma uric acid at 18 weeks of age was observed in heated compared to normal group. A very highly significant difference between genotypes, dwarfs ranking higher than normals, appeared in both groups, although the mean difference was less in heated group. As mentioned also in the same paper, plasma glucose at 18 weeks was slightly less in dwarfs than in normals and also in heated group compared to control group, although this was not significant. Plasma uric acid at 39 weeks of age, measured only in heated group, was also highly significantly different between genotypes, but this difference was comparatively less than at 18 weeks Sire family effect which was non significant at 18 weeks was highly significant for plasma uric acid at 39 weeks. From 18 to 39 weeks level of plasma uric acid increased but the increase in dwarfs was considerably lower than in normals. Increase of plasma uric acid with age has already been reported in the literature (BELL \& FreemanN, 1971).

Water intake in dwarfs was reduced by about 30 p. 100 in heated group in comparison with normals. As reduction in observed food consumption by dwarfs was 37 p. 100 , the ratio of water to food consumption was higher in dwarfs. On the other hand, dry matter in feces was significantly lower in dwarfs at $0.1 \mathrm{p} .100$ level while percent moisture in feces was significantly lower for this genotype at 1 p. 100 level. Genotype $\times$ family interactions for the latter two characters were also significant at 5 p. 100 level. There is an apparent discrepancy between the afore-mentioned higher ratio of water to food consumption, and the lower moisture percent in excreta for $d w$ hens. This discrepancy remains to be explained.

\section{B. Phenotypic correlations}

In the following, at the first place correlations are compared for dwarf and for normal hens. In addition, significant correlations which are common to both genotypes will be commented. Finally, possible effect of treatment on certain correlations will be discussed, but in view of the wide difference between the two environments for some traits like age at first egg, this will concern traits not associated with the former.

\section{a) Effect of genotype at the Dw locus on phenotypic correlations}

Tables 5 and 6 show that within each treatment a number of correlations are significantly heterogenous between the Dw and dw genotypes. For most of these correlations it is possible to pool environments and to obtain an unique estimate for each genotvpe. Part of these pooled estimates are no more significantly different between Dw and dw birds. In such cases, the meaning of a significant between genotypes heterogeneity found in only one environment is not clear and may be 
due to sampling, so that it is safe to wait for further data before proposing any interpretation.

Conversely, some correlation estimates remain significantly different between Dw and dw hens after pooling treatments (table 7).

The interpretation of differences between the dwarf and normal genotypes for most cases is not clear. The most clear-cut differences concern the trait, egg shell thickness and to some extent also shell percentage. Among normal-sized (Dw) hens, shell thickness is slightly negatively correlated with all traits concerning body weight, egg mass and egg number. On the contrary, for dw hens, there is a clear positive correlation with the same traits. The tendency, although less marked, is the same for shell percent. The same can be said for the correlations of egg shell thickness with plasma uric acid at 18 weeks of age, showing in both control and heated group a higher value for $\mathrm{dw}$ hens.

It may be suggested that for those dw hens, which have a lower food consump. tion on average, calcium is a more limiting factor than for their normalsized sisters, so that dwarfs with higher food intake (and correlated traits such as body weight and egg laying) are able to produce thicker shells. This seems to be confirmed by the fact that partial correlations of shell traits with either body weight, egg mass or egg number with fixed food intake among $d w$ hens are not significant : for instance, the corresponding values for egg shell thickness are respectively +0.05 , +0.14 and -0.07 (N.S.).

It does not seem that such positive correlations between egg shell traits and food intake and egg production were observed previously in dwarf stocks. The present result may be due to especially small size of dw hens in our Leghorn strain.

On the contrary, in a previous publication (MéRAT, 1969) in a medium-sized strain, a positive correlation was observed on dwarf hens and not on their normal counterparts, between egg production and adult body size. This was confirmed in more recent years on the same stock (unpublished data). This trend seems to be present here only in the control group, not in the «heated» group. However, in this last group delay in sexual maturity may have obscured this tendency.

Table 7 does not include traits which were measured only in one environment. Tables 5 and 6 show significant differences between genotypes at Dw locus for a few correlations concerning these traits. In the heated group, uric acid at 39 weeks of age is negatively correlated with egg shell thickness only for dwarfs. Water in feces shows among dwarfs positive correlation with egg and yolk weight, a negative one with total pause days, the corresponding correlations being low and of opposite signs for normals. Dry matter in feces shows a positive correlation with egg weight and shell thickness only for dwarfs. Finally, in the "control » environment, percentage of soft-shelled eggs shows correlations of opposite sign with egg weight for Dw and $\mathrm{dw}$ females. To date it is not possible to propose an interpretation of these differences.

\section{b) Significant correlations common to both genotypes and treatments}

A number of the observed correlations correspond to expected associations, e.g. of food intake with body weight, body weight gain, egg mass; of egg number with egg mass and of both with age at first egg, mean clutch length and pauses; of 
mean egg weight with shell thickness; of adult body weight with 18 -week body weight and body weight gain; and the negative correlation of mean egg weight with yolk/albumen ratio, corresponding to the known fact that large eggs have a smaller proportion of yolk.

On the other hand, as the main purpose of this paper concerns effects associated with the sex-linked dwarf gene, we shall limit further the discussion of significant correlations (as common estimates to both genotypes and treatments) to those which to our knowledge have not been mentioned previously in the literature. On the whole body weight is positively correlated with clutch length and negatively with pauses. This may be due to the small size of the strain used here, including Dw birds. To a lesser extent, the trend is the same for body weight gain. Of interest are also the correlations of uric acid in plasma at 18 weeks of age : pooling genotypes and treatments, the correlation (based on 187 d.f.) is -0.21 with egg number ( $\mathrm{p}<0.01$ ) and -0.20 with mean clutch length $(\mathrm{p}<0.01)$. This fact is the more interesting as this blood parameter is measured before sexual maturity, and so is not a mere indicator of laying rate. It remains to be verified if earlier measurements of uric acid in plasma could be used as an aid for early selection for egg production.

A few significant correlations concern traits measured only for one treatment. This is the case for per cent double yolked eggs in the control group (negative correlation with yolk/albumen ratio and egg shell thickness); for per cent softshelled eggs (negative with egg shell thickness, as found by Silber \& MÉrat, 1974). In the heated group, uric acid in plasma at end of laying recording is negatively correlated with body weight, weight gain, egg mass, food intake, shell percent, and rectal temperature : these correlations are largely different from those observed for plasma uric acid at 18 weeks, which is in agreement with the low correlation which appears between plasma uric acid measurements at the two ages. Also in heated group, dry matter in feces shows a positive correlation (as might be expected) with food intake, and with body weight and egg mass. Its correlation with $\mathbf{R}$ ( residual food intake ») is positive but low and not significant.

\section{c) Correlations differing between treatments}

A number of correlations differ between environments only by their value but not by their sign. Those differing also by their sign are generally significantly different from zero in one environment and of opposite sign but insignificant in the other environment. Among them, those concerning age at first egg are difficult to interpret, in view of the very large difference between treatments for this trait. On the other hand, the following deserve to be mentioned : percent egg shell with feed intake, egg mass, mean clutch length (positive in heated group, slightly negative in control) and with pauses and uric acid at 18 weeks (negative in heated group); rectal temperature with egg mass and egg number (negative in control group) and pauses (positive in control group).

\section{Conclusion}

The present study was aimed to contribute to the problem of possible utilization of the sex-linked dw gene for egg production. In this respect, it seems safe to 
conclude from our results that introduction of the dwarf gene for such use cannot take place into a population already of too small size such as was our Leghorn strain. This is in agreement with the positive correlation between egg laying and adult body size that we observed previously in small size dwarf strain (Mérat, 1969 and more recent inpublished data) and also with the positive correlation shown in the present paper in $\mathrm{dw}$ birds between body size or feed intake on one hand and egg shell thickness or percent on the other hand. This too small size of the stock used here may also contribute to explain the absence of any clear advantage of $\mathrm{dw}$ hens for egg laying traits at higher temperature in our experiment, contrary to a slight advantage observed in previous results.

However, it may be noticed that in spite of the rather poor egg laying overall performance of dwarf hens in this strain (especially in the conditions which occurred in « heated » treatment) food efficiency of dwarfs as measured by the ratio of food consumed to egg mass produced, was not worse than for their normal full- or half sisters. This conclusion would be reinforced if the lower proportion of abnormal or cracked eggs (at least at normal temperature) is taken into account.

Finally our data may show that the main problem for possible use of dwarf egg laying stock would be to improve rate of lay, especially mean clutch length which is much lower among dwarf layers than for $D w$ hens. Selection on this last trait or on time interval between successive ovipositions can be suggested. It remains open to question if additional traits would be useful as an aid to such selection. The negative phenotypic correlation observed between plasma uric acid at 18 weeks of age (before the onset of laying) with clutch length and egg number may deserve further observations, to know if these correlations are present also at the genetic level and if they are found in other populations.

\section{Received for publication in March 1982.}

\section{Acknowledgements}

We would like to thank $\mathrm{D}^{\mathrm{r}}$ F. Ricard (Station de Recherches avicoles, 37380 Nouzilly) for his very useful criticisms to this manuscript.

\section{Résumé}

Gène de nanisme lié au sexe (dw) dans une lignée de pondeuses Leghorn blanches à température normale ou élevée

Des poules Leghorn blanches, issues de 7 familles de pères en ségrégation au locus de nanisme lié au sexe ( $\mathrm{Dw}$ et $\mathrm{dw})$, ont été contrôlées en cages individuelles à température modérée (lot témoin, 13 à $23^{\circ} \mathrm{C}$ ) ou élevée (lot chauffé, 25 à $34^{\circ} \mathrm{C}$ ) de 18 à 39 semaines d'âge. Les mesures enregistrées concernent le poids corporel, l'efficacité alimentaire, la production d'œufs jusqu'à la $38^{\mathrm{e}}$ semaine d'âge, des facteurs de qualité des œufs, l'acide urique et le glucose plasmatique à 18 semaines. Dans le lot "chauffé », l'acide urique plasmatique à 39 semaines, la consommation journalière d'eau, la matière sèche et le pourcentage d'eau des déjections, ont également été mesurés. Les résultats sont les suivants : 
1. Le poids corporel adulte chez les naines était de 38 p. 100 inférieur à celui des normales dans le lot témoin et 32 p. 100 dans le lot chauffé, alors que la consommation alimentaire des poules naines était respectivement de 36 et 37 p. 100 inférieure en lot témoin et en lot chauffé.

2. Le nombre d'œufs pondus entre le $1^{\text {er }}$ ouf et 39 semaines d'âge chez les naines était de 20 et de 41 p. 100 inférieur à celui des poules de taille normale respectivement en lot témoin et en lot chauffé. La masse totale d'œufs produite par 28 jours chez les premières présentait une réduction de 34 p. 100 par comparaison aux normales dans le lot témoin et de 37 p. 100 dans le lot chauffé. Le rapport du poids moyen des œufs des naines à celui des normales augmentait de 0,85 à 0,89 de la $27^{\circ}$ à la $39^{\circ}$ semaine d'âge (lot témoin). Durant les 7 premières semaines de ponte, le pourcentage d'œufs à deux jaunes, sans coquilles ou fêlés (observé en lot témoin seulement) était très significativement inférieur chez les poules naines. Dans les deux groupes, la taille moyenne des séries de ponte était significativement plus faible pour ces dernières. Par contre, la durée totale des "pauses» n'était pas significativement plus élevée pour le génotype dw.

3. L'âge à maturité sexuelle des naines n'était retardé que d'un jour (non significatif) par rapport aux normales dans le groupe témoin. Dans le lot chauffé, un retard inhabituel était observé tant chez les naines que chez les normales (201 et 182 jours en moyenne respectivement pour les deux génotypes). Ceci ne paraît pas être dû uniquement à la température.

4. L'épaisseur des coquilles était inférieure chez les poules naines dans les deux environnements, la différence étant plus élevée et hautement significative en lot chauffé.

5. Un effet hautement significatif du génotype était observé pour la température rectale à la fois dans le lot témoin et le lot chauffé. La valeur de ce paramètre était inférieure pour les poules naines comparées aux normales.

6. Un effet hautement significatif du génotype et de la famille de père était observé pour le taux d'acide urique plasmatique mesuré à 39 semaines en lot chauffé. La différence entre génotypes était de même sens (valeur plus élevée pour les poules $\mathrm{dw}$ ) mais comparativement moindre que celle observée à 18 semaines. Dans l'ensemble, le taux d'acide urique du plasma à 39 semaines était augmenté par rapport à la valeur obtenue à 18 semaines.

7. Les corrélations phénotypiques du pourcentage ou de l'épaisseur de coquille avec le poids corporel, la consommation d'aliment, la masse d'œufs, le nombre d'œufs pondus et la taille des séries de ponte étaient toutes positives et pour la plupart significatives chez les naines; chez le génotype $\mathrm{Dw}$, elles étaient non-significatives et généralement négatives.

8. En général, l'acide urique plasmatique à 18 et 39 semaines était en corrélation négative avec le nombre et la masse d'œufs et la taille des séries de ponte. Ces corrélations semblaient plus accentuées en lot témoin et pour le génotype Dw.

9. La réduction spécialement importante du poids corporel et des performances de ponte et l'abaissement des caractéristiques de coquille associés au gène dw peuvent correspondre à la petite taille de la population Leghorn utilisée dans l'étude présente.

\section{References}

DE ANDrade A.N., Rogler J.C., Featherston W.R., 1976. Influence of constant elevated temperature and diet on egg production and shell quality. Poult. Sci., 55, 685-693.

Arscott G.H., Bernier P.E., 1968. Effect of dietary protein on performance of dwarf White Leghorn layers. Poult. Sci., 47, 1652 (abstr.).

Banerjee A.K., Bordas A., Mérat P., 1981. The effect of temperature and genotype on growth traits, plasma glucose and uric acid in Dwarf and Normal White Leghorn females. Ann. Génét. Sél. anim., 13, 255-268.

Bell D.J., Freeman B.M., 1971. Physiology and biochemistry of the domestic fowl. Vol. 2, pp. 924-925. Academic Press, London and New York.

Bernier P.E., Arscott G.H., 1972. Fifteen years of observations on the dwarf gene in the domestic fowl. Ann. Génét. Sél. anim., 4, 183-215. 
Bordas A., Mérat P., 1976. Effect of laying on food and water intake in dwarf and normal hens. Br. Poult. Sci., 17, 415-426.

Bordas A., Mérat P., 1981. Genetic variations and phenotypic correlations of food consumption of laying hens corrected for body weight and production. Br. Poult. Sci., 22, 25-33.

BYERLY T.C., 1941. Feed and other costs of producing market eggs. Bull. University of Maryland Agricultural Experimental Station A 1.

CARMon L.G., Huston T.M., 1965. The influence of environmental temperature upon egg components of domestic fowl. Poult. Sci., 44, 1237-1240.

Doran B.H., Quiseneerry J.H., 1974. Performance of normal and dwarf birds fed two levels of protein during growing and laying periods. Poult. Sci., 53, 1919-1920.

Dorminey R.W., Arscott G.H., Bernier P.E., 1973. Effect of thyroprotein feeding on performance of Dwarf White-Leghorn pullets. Poult. Sci., 52, 1924-1932.

French H.L., Nordskog A.W., 1973. Performance of Dwarf chickens compared with normal small bodied chickens. Poult. Sci., 52, 1318-1328.

Gleichauf R., 1973. The use of the dwarfing gene, dw, in broiler production. 4th European Poultry Conference, London, 543-549.

Gous R.M., Byerly T.C., Thomas O.P., Kessler J.W., 1978. A partition equation to predict food and energy intake by laying hens. 16th World's Poultry Congress, Malta, 1-8.

Guillaume J., 1969. Consequences de l'introduction du gène de nanisme dw sur l'utilisation alimentaire chez le poussin femelle. Ann. Biol. anim. Biochim. Biophys., 9, 369-378.

Guillaume J., 1975. Thesis Doct. ès Science, Univ. of Tours, France, cited by Guillaume J., 1976, World's Poult. Sci. J., 32, 285-304.

Guillaume J., 1976. The dwarfing gene dw : its effects on anatomy, physiology, nutrition, management. Its application in poultry industry. World's Poult. Sci. J., 32, 285-304.

Horst P., Petersen J., 1978. Der effekt des dwarf-genes auf das akklimatisationsvermögen von legehennen an hole umwelttemperaturen. 16th World's Poultry Congress, vol. I, 247-254.

HutT F.B., 1959. Sex-linked dwarfism in the fowl. J. Hered., 50, 209-221.

JaAP R.G., Mohammadian M., 1969. Sex-linked dwarfism and egg production of broiler dams. Poult. Sci., 48, 344-346.

LECLERCQ B., BLUM J.C., 1975. Restricted feeding of growing pullets : interaction between the method of restriction and the dwarfism gene (dw). Archiv. Geflügelk., 39, 153-158.

Leeson S., Lewis D., Shrimpton D.H., 1973. Multiple linear regression equations for the prediction of food intake in the laying fowl. Br. Poult. Sci., 14, 595-608.

Lillie R.J., Ota H., Whitehead J.A., Frobish L.T., 1976. Effect of environment and dietary energy on caged Leghorn pullet performance. Poult. Sci., 55, 1238-1246.

Mérat P., 1969. Etude d'un gène de nanisme lié au sexe chez la poule. I - Description sommaire et performances. Ann. Génét. Sél. anim., 1, 19-26.

MÉRAT P., 1970. Biometric characters and maternal effects associated with a gene tor dwarfism in the hen. XIV World's Poultry Congress, Madrid, 283-287.

Mérat P., 1972. Quelques effets du gène dw sur la ponte et sur la qualité des œufs. Ann. Génét. Sél. anim., 4, 217-223.

Mérat P., Guillaume J., 1969. Etude d'un gène de nanisme lié au sexe chez la poule. II - Fonctionnement thyroïdien. Ann. Génét. Sél. anim., 1, 131-133.

Mérat P., Bordas A., Lefebvre J., 1974. Effets associés aux gènes dw (nanisme) et Na ( « cou nu») chez la poule sur la production d'œufs et la consommation alimentaire à deux températures. Ann. Génét. Sél. anim., 6, 331-343.

Mérat P., Bordas A., Ricard F.H., 1980. Composition anatomique, production d'œufs et efficacité alimentaire de poules pondeuses. Corrélations phénotypiques. Ann. Génét. Sél. anim., 12, 191-200.

McDonald D., 1978. Feed intake of laying hens. World's Poultry Sci. J., 34, 209-221. 
Mohammadian M., JaAP R.G., 1972. Effect of the sex-linked dw Dwarfing gene on body growth of chickens. Poult. Sci., 51, 1701-1707.

Polkinghorne R.W., Lowe A.G., 1973. A comparison of Dwarf and normal crossbred layers. J. austr. Inst. agric. Sci., 39, 77-78.

Prod'homme J., Mérat P., 1969. Etude d'un gène de nanisme lié au sexe chez la poule. III - Consommation alimentaire et production suivant la teneur en calcium de la ration. Ann. Génét. Sél. anim., 1, 135-145.

Quisenberry J.H., 1972. High density diets for Dwarf layers. Ann. Génét. Sél. anim., 4, 271-279.

Quisenberry J.H., Bradley J.W., 1971. Response of midget birds to space and dietary limitations. Poult. Sci., 50, 1621.

RAPP K.G., 1970. Die Wirtschaftlichkeit einer Zwerg mutante der weissen Leghorn in der Linien- und Hybridzucht. Diss. Univ. Göttingen.

Reddy P.R.K., Udale R.W., Siegel P.B., 1974. Growth and reproduction of Dwarf and normal sibs in diverse genetic backgrounds. Poult. Sci., 53, 1970-1971.

RiCARD F.H., 1972. Croissance et caractéristiques de carcasse de poulets issus de mères normales ou naines. Ann. Génét. Sél. anim., 4, 173-182.

RiCARd F.H., Cochez L.P., 1972. Le gène de nanisme dw et les performances des poules dans une souche de type femelle-chair. Ann. Génét. Sél. anim., 4, 297-303.

Selvarajah T., 1974. Personal communication, cited by Guillaume J., 1976. World's Poultry Sci. J., 32, 285-304.

Silber J., Mérat P., 1974. Genetic study of the laying of soft-shelled eggs in the fowl. $X V$ World's Poultry Congress, New-Orleans, 491-493.

Summers J.D., 1972. Nutrition of the dwarf layers. Ann. Génét. Sél. anim., 4, 251-258.

VAN MiddLEKOOP J.H., 1973. Influence of the dwarfing gene on yolk production and its consequences for normal egg laying of White Plymouth Rock pullets. Arch. Geflügelk., 37, 192-196.

Yamada Y., Watanabe K., Ebisawa S., Futamura K., 1972. Effect of the sex-linked Dwarf gene on performance of broiler dams. Jap. Poultry Sci., 9, 286-290.

Zlochevskaya K., Penionzhrevich E., 1973. Dwarfing genes and body proportions of meat and egg type hens. 4th European Poultry Conference, London, 537-572. 